\title{
A Computational System for Investigating Chemotaxis-Based Cell Aggregation
}

\author{
Manolya Eyiyurekli ${ }^{1} \quad$ Peter I. Lelkes ${ }^{2} \quad$ David E. Breen ${ }^{1}$ \\ ${ }^{1}$ Department of Computer Science, College of Engineering \\ ${ }^{2}$ School of Biomedical Engineering, Science and Health Systems \\ Drexel University, Philadelphia PA 19104, USA
}

\begin{abstract}
We have developed a software system that simulates chemotaxis-based cell aggregation in $2 \mathrm{D}$. The model implemented within the system consists of such cell behaviors as chemical diffusion/detection, motility, proliferation, adhesion and life cycle stages. Each virtual cell detects the state of the environment, and responds to the environment based on a pre-defined "program" and its own internal state. Cells are discrete units that are located on a grid, exist in discrete states (e.g. active or dying) and perform discrete tasks (e.g. divide and attach), but they also contain and are affected by continuous quantities (e.g. chemoattractant concentrations, gradients, age and velocities). This paper provides an overview of our chemotaxis-based aggregation model and details the algorithms required to perform chemotaxis-based cell aggregation simulation. A number of biological studies are being conducted with the system. They include fine-tuning the model parameters to reproduce in vitro $\mathrm{PC} 12$ cell aggregation experiments and parametric studies that demonstrate the effect that the model's components have on cell aggregation dynamics.
\end{abstract}

\section{Introduction}

Chemotaxis (CTX) is the phenomenon where cells detect gradients of chemicals (growth factor, cytokine) and respond to the chemical stimulus by moving either towards (positive CTX) or away (negative CTX) from the source. Multicellular aggregates and eventually tissue-like assemblies are formed when individual cells attach to each other and when this aggregation leads to subsequent cellular differentiation. Understanding the influence of the many components of CTX on overall cell aggregation should lead to a more detailed understanding of the mechanisms involved in tissue assembly and organogenesis, and also facilitate the development of novel technologies for tissue engineering based on controlling or directing these underlying biological processes. Modeling cell aggregates and their assembly/differentiation into functional tissues has implications for the mechanistic understanding of this process in vivo, as well as for "in vitro embryology". Beyond tissue engineering, chemotaxis also plays a significant role in a number of other biomedical phenomena of great interest, e.g. tumor formation [1] and bacteria accumulation [2]. Chemotaxis is also currently being explored as a central modeling paradigm for self-organizing geometric primitives that automatically produce user-defined shapes.

Given the important role that chemotaxis plays in a variety of biological processes, we have developed a software system that simulates chemotaxis. The system provides a computational testbed for investigating the dynamics of chemotaxis and the biological components/interactions that affect and control it. While we are ultimately interested in modeling chemotaxis in 3D, our initial computational system is capable of simulating CTX-based cell aggregation in 2D. The model implemented within the system consists of the cell behaviors needed for aggregation, such as chemical diffusion/detection, motility, proliferation, adhesion and life cycle stages. An aggregation simulation is performed by modeling individual cells existing in an environment that contains a diffusing 
chemoattractant chemical. Each cell detects the state of the environment, and responds to the environment based on a pre-defined "program" and its own internal state. The model is hybrid in nature as it includes both discrete and continuous components. The cells are discrete processing units that are located on a grid, exist in discrete states (e.g. active or dying) and perform discrete tasks (e.g. divide and attach), but they also contain and are affected by continuous quantities (e.g. chemical concentrations, gradients, age and velocities). This paper provides an overview of our model, and details the algorithms required to perform chemotaxis-based cell aggregation simulations.

\section{Previous Work}

The most relevant research in cell modeling and simulation may be placed in two broad categories, 2D grid-based models, and hybrid models of discrete cells with continuous motions/quantities.

2D and 3D grid based models focus on inter-cellular processes such as signaling, motion and attachment to an extracellular matrix. Some of these models also combine the internal workings of individual cells with external processes. These models treat cells as particles collectively moving on a rectilinear grid. The Cellular Potts Model (CPM) [3], a lattice-based stochastic framework, has been used to model adhesion driven motion created by varying surface energies of different kinds of cells in order to simulate cell sorting. CPM has been used as a part of other models to simulate the rearrangement of cells during morphogenesis $[4,5]$ and to model avascular tumor growth [6]. A discussion of how cell-centered simulations like CPM can help to explain aspects of developmental biology can be found in [7]. Some models in this category [8-10] use cellular automata $[11,12]$ to model cell motility and differentiation in order to simulate tumor growth $[13,14]$ and embryogenesis [15].

There are other models that use a 2D-grid based approach, combined with environmental forces, to simulate the deformation and aggregation of flowing red blood cells [16]. Monte-Carlo models, non-deterministic techniques that produce solutions by random assignments to uncertain model variables, using only Brownian Motion [17] and a combination of Brownian Motion and cellular automata [18] have been developed to simulate tumerogenesis.

The latter approach utilizes hybrid models that contain both discrete and continuous components. These models define cells as independent, discrete units that can move in response to continuous forces and influences. This approach has found use in simulating multicellular pattern formation [19,20], studying the chemotactic motility of individual cells [21,22] and the computational modeling of cell adhesion [23]. Our model simulates chemotaxis, where chemical-based cell-cell signaling is the main source of communication. A similar use of cell-cell signaling can be found in [21], which investigated cell motility with respect to chemotactic forces. Some models integrate two or more of these approaches in one simulation system. COMPUCELL-2D [24] and 3D [25] are two simulation systems that have been developed with both CPM and diffusion-based continuum models They are able to simulate signaling and cells moving in response to adhesion forces. Our model clearly falls into the second category of hybrid models. While it contains many of the elements from previous efforts [19-21,24,25], such as the discrete cells that move, divide and attach, our approach also includes a more detailed modeling of proliferation and apoptosis.

\section{Model Overview}

In our simulation system each cell is defined by a collection of physiologically relevant parameters and actions [26], such as the number and position of chemical receptors on the cell surface, location of the cell, age, life cycle stage, chemoattractant emission and response rates, diffusion radius, 


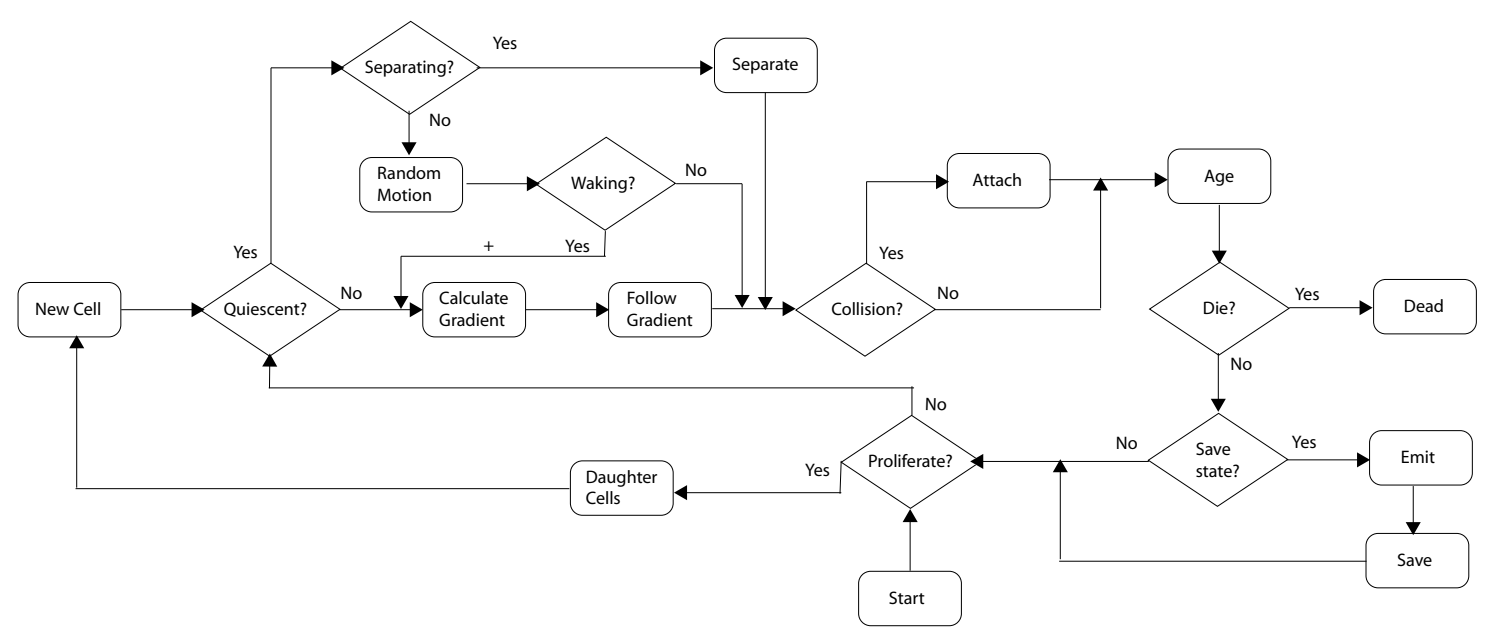

Fig. 1. Computational flow of cell aggregation simulation per time step per cell.

proliferation rate, quiescent period, time of apoptosis, and number of attached cells. Our virtual cells are able to emit chemoattractants, sense the chemoattractant gradient, move in the direction of the gradient, proliferate, adhere to other cells, age and die.

A cell's life cycle and behaviors are implemented as set of actions that are performed during each time step of a simulation. A single aggregation simulation is comprised of a series of these time steps. A cell's actions for each one of these time steps are outlined in Figure 1. Newly proliferated cells remain in a quiescent state for a user-defined period. These cells are not Active and cannot divide. A quiescent cell can perform three types of actions. Immediately after cell division a new daughter cell separates from its parent. It moves with a constant velocity along the division axis for a user-defined period of time. Upon completion of this separation period the cell begins to respond to the chemoattractant gradient in its local environment. During the Waking phase there is some randomness in the cell's movement. These random motions vanish as the cell becomes Active. Based on the chemical fields produced by nearby cells a gradient is calculated and the cell/aggregate moves in the gradient direction in response to it. A cell is capable of attaching to other cells upon collision. A cell's age is incremented at each time step. If the cell is apoptotic, it probabilistically determines if it should die. If the state of the complete environment is to be saved for visualization purposes, the cell emits and stores its chemical field in the chemoattractant array. If in the Active stage, the cell probabilistically determines if it is time to divide. If it divides, its and its daughter cell's age are set to zero, and they enter the quiescent stage.

\section{System Description}

The simulation system is implemented in $\mathrm{C}++$ using the OpenGL library. It consists of two classes with a total of 2300 lines of code. The cell class contains the variables (see Table 1) and methods (see Figure 1) which define a virtual cell. The main class implements the simulation system that performs the simulation with a number of cells. Simulations were run on a 64-bit linux cluster running Dual-Opteron nodes with 1GB of RAM, and Gentoo 2.6. The simulation execution times mostly depend on the number of cells and discrete time steps in the simulation. The average running 
Table 1. Virtual cell variables

\begin{tabular}{ll}
\hline Variable & Description \\
\hline ID & Cell's unique identifier \\
Coordinates[2] & 2D grid coordinates defining the location of the cell \\
Age & Age of the cell \\
lifeCycleStage & One of: Active, Quiescent, Waking, Dying, Dead \\
AggregateNumber & ID number of the cell's aggregate \\
Sensors[8] & Chemoattractant concentration read at sensors \\
Gradient [2] & Stores the gradient vector \\
dieFlag & Cell dies if set to 1 \\
divideFlag & Cell divides if set to 1 \\
NumberOfNeighbors & Number of attached cells \\
\hline
\end{tabular}

time for a 24-hour simulated aggregation experiment with approximately 1,500 cells and 1440 time steps $(\Delta t=1$ minute $)$ is approximately $30 \mathrm{CPU}$-minutes.

Initialization and Main Loop. A cell aggregation simulation is initialized by reading in model parameters from the command line, and randomly placing cells and aggregates in the simulation environment, as described in Algorithms 1 and $2^{1}$. The number of cells and aggregates at the beginning of a simulation run can be based on aggregate size distribution data derived from in vitro experiments, or can be defined by the user. The cell/aggregate distribution information (stored in the file SizeDistribution.txt), i.e. the number of singles cells, two-cell aggregates, etc., is used to set the initial conditions for the simulations, and determines how many aggregates of the varying sizes should be defined at the beginning of the simulation. At initialization time cells/aggregates are randomly placed in non-intersecting locations in the simulation environment (see Algorithm $2^{1}$ ). Prior to cell placement, a number of aggregate configurations, i.e. specific descriptions of cell groupings and connections, for each aggregate size present in the experiments are pre-calculated. The aggregate configurations are created by performing several aggregation simulations and recording the geometry of the resulting aggregates for the different sizes. The aggregate configurations are stored in files, each containing several random arrangements. Each aggregate is represented by the number and coordinates of neighboring cells for each of its cells. During initialization the aggregates are read from the configuration files and placed in the simulation environment. The main loop, detailed in Algorithm $3^{1}$, Figure 1 and Section 3, is then executed to activate the virtual cells.

Proliferation. All non-quiescent and non-apoptotic cells may probabilistically divide, with their proliferation probabilities being a function of the number of cells attached to them. See Algorithm $4^{1}$. Cell attachments increasingly inhibit proliferation. A cell's proliferation probability is decreased by a factor of $e^{-(n+1)}\left(K_{3}\right.$ in Algorithm $\left.4^{1}\right)$, where $n$ is the number of cells attached to the cell. Once a cell divides the ages of both daughter cells are set to zero, and they enter the quiescent state. After division a new cell is randomly placed in an open location next to the original. With probability $P_{\text {Separation }}$ the new cell separates from the original cell, otherwise it stays attached.

\footnotetext{
1 The detailed pseudocode for the algorithms is located at http://www.cs.drexel.edu/ david/Papers/ECAL07_Algs.pdf. The algorithms are included in the submission as an appendix, but will only be available on the web, if the paper is published.
} 

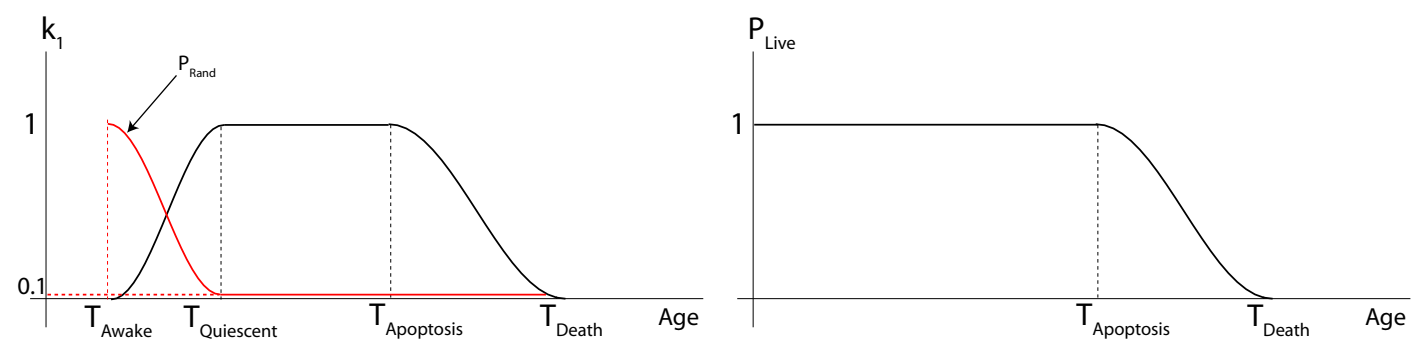

Fig. 2. Left: Change in chemoattractant emission and response as a function of age $\left(k_{1}\right)$ and probability of taking a random step $\left(P_{\text {Rand }}\right)$. Right: Probability of being alive as a function of age.

Quiescence. After a cell divides it enters the quiescent phase, a period where the cell's mechanisms and behaviors gradually become functional. If cells separate after a single cell division the two daughter cells move away from each other with a constant velocity along the division axis for a user specified time period $\left(T_{\text {Separation }}\right)$. If the dividing cell is attached to an aggregate, only the new cell possibly separates. Newly created cells do not immediately contribute to or respond to the chemotactic field around them . Halfway through the quiescent period $\left(\left(T_{\text {Awake }}=T_{\text {Quiescent }} / 2\right)>\right.$ $T_{\text {Separation }}$ ), they start emitting and sensing chemoattractants with an increasing rate (see Figure 2: Left). After the separation period $\left(T_{\text {Separation }}\right)$ cells randomly move. After $T_{\text {Awake }}$ cells are increasingly affected by chemoattractant gradients. The probability that a quiescent cell takes a random step $\left(P_{\text {Rand }}\right)$ decreases as it becames more awake. This probability becomes $1 \%$ for fully active cells. Conversely waking cells follow the chemoattractant gradient with probability $1-P_{R a n d}$. See Algorithm $6^{1}$ for more details.

$$
\text { Velocity }=\lambda * k_{1} * \nabla C,
$$

Cell/Aggregate Movement. An unattached cell moves in the direction of the chemoattractant gradient with a velocity proportional to the gradient, with $\lambda$ being the proportionality constant. A cell's chemotactic response is also affected by the cell's life cycle stage and age. This feature is implemented with scale factor $k_{1}$. See Figure 2:Left and Equation 1. If a cell is part of an aggregate, its gradient-based velocity is combined with the velocities calculated for the other cells in the aggregate to produce an average velocity for the whole aggregate. An aggregate's velocity is also inversely proportional to its mass, i.e. the number of its constituent cells. The algorithms that implement cell/aggregate movement are detailed in Algorithms 6 and $7^{1}$.

At every time step and for each cell, the surrounding cells that can influence the current cell are identified and added to the cell's neighbor set. Looping through every cell, we calculate the distance from the current cell to every other cell. If the distance between the two is less than the other cell's $R_{\text {Max }}$ value, the other influencing cell is added to the current cell's neighbor list. $R_{\text {Max }}$ is the maximum distance at which a cell's chemoattractant concentration can be detected by another cell, and is a function of the cell's chemoattractant emission rate, $C_{0}$. We perform this check for both cells and update both cells' neighbor list in order to minimize computations. Once we determine which cells' chemical fields may affect the current cell, the chemoattractant concentration emitted from each of the influencing cells is calculated at the eight receptor sites on the current cell's surface 
$\left(\right.$ See Algorithm $\left.5^{1}\right)$, with the equation

$$
C(r)=\frac{k_{1} * C_{0}}{1+r}
$$

where $C_{0}$ is the chemical concentration at the emitting cell's surface, and $r$ is the distance between the emitting cell's surface and the sensing cell's receptor. The overall chemical gradient sensed by a cell is calculated with the following equations,

$$
\begin{array}{cc}
C_{x}=\frac{\Lambda_{2}-\Lambda_{6}+\frac{\Lambda_{1}-\Lambda_{5}-\Lambda_{7}+\Lambda_{3}}{\sqrt{2}}}{2 * r_{c}} & C_{y}=\frac{\Lambda_{8}-\Lambda_{4}+\frac{\Lambda_{7}+\Lambda_{1}-\Lambda_{5}-\Lambda_{3}}{\sqrt{2}}}{2 * r_{c}} \\
\nabla C=\left(C_{x}, C_{y}\right) & \Theta(\nabla C)=\operatorname{atan}\left(C_{y} / C_{x}\right),
\end{array}
$$

where $\Lambda_{i}$ is the chemical concentration calculated at receptor $r_{i}, r_{c}$ is the cell's radius, and $\Theta$ is the angle used to determine the cell's displacement direction. The locations of the numbered receptors are provided in Figure 3:Left. Cells do not self-stimulate nor are they influenced by the cells in their aggregate, i.e. cells in the same aggregate are not added to the neighbor list.

In an effort to simplify movement, collision, attachment and cell division computations, we discretize the environment in which the cells exist and move into a hexagonal grid with a toroidal topology. While each cell contains a Cartesian location $P_{C a r}$, having the cells positioned in a hexgrid ensures equal distances between each grid point and its six neighboring grid points. The toroidal topology connects the left side of the finite environment to the right side, and the top edge to the bottom edge. So as cells move over an edge in the underlying grid they are placed at the opposite side of the grid. The conversion from hex-grid coordinates $P_{H e x}(\mathrm{i}, \mathrm{j})$ to Cartesian coordinates $P_{C a r}$ $(\mathrm{x}, \mathrm{y})$ is given in Equation 4, where $d$ is the distance between grid-points ( $1 \mu \mathrm{m}$ for our simulations).

$$
P_{C a r}=\left[\begin{array}{ll}
d & \frac{1}{2} d(j \bmod 2) \\
0 & \frac{\sqrt{3}}{2} d
\end{array}\right] \cdot P_{H e x}
$$

A cell therefore can only move in one of the six directions as seen in Figure 3:Right. Cells and aggregates move in the direction which is closest to its velocity vector. The closest direction is determined by the identifying the smallest angle between the six potential discrete directions and the cell's continuous velocity vector.

The minimum distance a cell can move on the hexagonal grid is $1 \mu \mathrm{m}$. It is possible that the displacement $\delta$ produced by a particular chemoattractant gradient and cell/aggregate mass is less than $d$. When this occurs, $\delta$ is stored and accumulated until the length of the accumulated vector is greater than $d$. At that point the cell/aggregate is moved. It is also possible that $\delta$ is not a multiple of $d$, i.e. $\delta=a * d+b, b<d$. In this case the cell/aggregate is moved $a$ steps and the amount $b$ is saved for future accumulation. It is also possible that a cell's displacement is more than $d$ in a defined $\Delta t$. In this case the simulation time step is divided into smaller periods so that the maximum displacement over one period is $d$; thus facilitating accurate collision detection.

Collision Detection and Attachment. Collisions are detected by checking if any two cell centers are within a distance of $2 *$ Radius. Each cell pair is only tested once. Once a collision is detected three scenarios are possible: (See Algorithm $8^{1}$.)

- both cells are singles, and they form a doublet; 

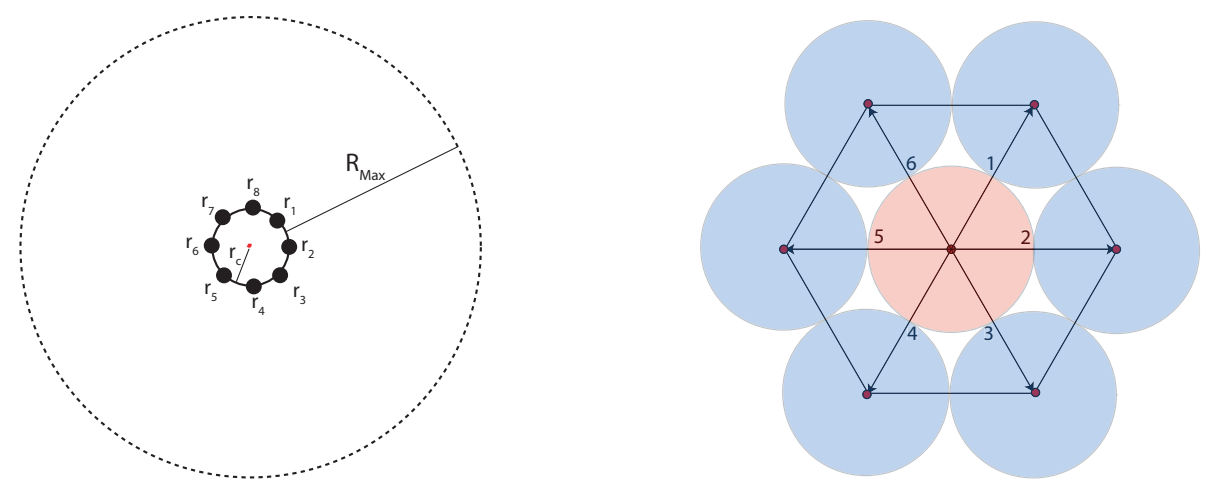

Fig. 3. Left: A single cell has radius $r_{c}$ and has eight chemoattractant receptors identified as $r_{i}$. The cell's chemoattractant cannot be sensed past $R_{M a x}$. Right: The cell at the center can move in one of the six directions defined by the hexagonal grid.

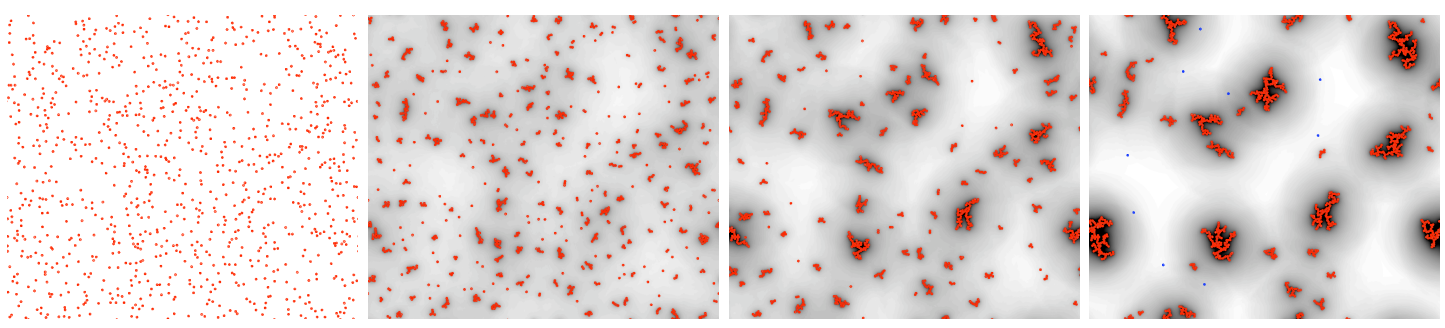

Fig. 4. Results from an in silico cell aggregation experiment. Time $=0$ hr, 6 hrs, 15 hrs and 25 hrs.

- one of the cells is single, the other belongs to an aggregate, and the single cell joins the aggregate;

- both cells belong to aggregates, and all of the cells in one aggregate join the other. The aggregate with the smaller mass joins the larger aggregate to save computation time.

Aging. Each cell has an age which is initially set to zero, and is incremented at every time step. The Age algorithm (See Algorithm $9^{1}$ ) sets a cell's life cycle state. Cells are initially in a Quiescent state. After $T_{\text {Quiescent }}$ they enter a fully active state. If a cell is still unattached after $T_{\text {Apoptosis }}$ it enters Dying state and has an increasing probability of dying. (See Figure 2:Right). Once a cell dies it is removed from the CELLS list and is no longer processed.

Visualization. It is possible to produce images that capture the changing state of an aggregation simulation. When generating these images every active cell emits chemoattractants into the environment. A 2D Cartesian grid is used to store the chemoattractant concentration. The amount of chemoattractant concentration at a grid point is calculated from the diffusion function (Equation 2) and added to the grid point. (See Algorithm $10^{1}$ ). Since chemical concentrations are emitted into the chemoattractant grid only for visualization purposes, these values are calculated at a user-specified time interval different than the simulation time step. For example, while the simulation time step may be 1 minute, an image capturing the locations of cells and the chemoattractant concentration distribution in the environment may only be generated and saved (using the SaveState() routine) once every simulation hour. See Figure 4 for example output images. 
Table 2. Model parameter values that produce the optimal fit to the PC12 in vitro results in Figure 5:Right.

\begin{tabular}{lll}
\hline Parameter & Description & Value \\
\hline$\Delta t$ & Simulation time step & $1 \mathrm{~min}$ \\
$d$ & Grid discretization & $1 \mu \mathrm{m}$ \\
Dim & Grid dimension & $3250 \times 3250\left(3250 \mu \mathrm{m}^{2}\right)$ \\
$C_{0}$ & Chemoattractant concentration at cell surface & 90 molecules $/ \mu \mathrm{m}^{2}[27]$ \\
$R_{\text {Max }}$ & Maximum radius of influence & $100 \mu \mathrm{m}[28]$ \\
$r_{c}$ & Cell radius & $6 \mu \mathrm{m}[29]$ \\
$T_{\text {Apoptosis }}$ & Start time of apoptosis & $18 \mathrm{~h}[30]$ \\
$T_{\text {Death }}$ & Time when all single cells have died & $24 \mathrm{~h} \mathrm{[30]}$ \\
$P_{\text {Div }}$ & Probability of cell division per time step & 0.07 \\
$P_{\text {Separation }}$ & Probability of separation after cell division & 0.20 \\
$T_{\text {Separation }}$ & Separation time after cell division & $20 \mathrm{mins}$ \\
$T_{\text {Quiescent }}$ & Quiescence end time & $90 \mathrm{mins}$ \\
$\lambda$ & Chemotaxis response factor & 0.055 \\
\hline
\end{tabular}

\section{$5 \quad$ Results}

Our cell aggregation simulation system is currently being employed for a number of computational experiments. In order to validate our model and computational system we have performed an in vitro PC12 aggregation experiment. The in vitro experiment was imaged [31], and the aggregate size distribution at the start and end of the experiment were extracted from the images. We then fine-tuned our model and its parameters to recreate in silico the aggregation distribution that was produced in the in vitro experiment. See Figure 5 for a sample image from the in vitro experiment and the histograms containing the resulting aggregate size distributions from the in vitro and in silico experiments. See Table 2 for the optimal model parameters. The last five parameters $\left(P_{\text {Div }}\right.$, $\left.P_{\text {Separation }}, T_{\text {Separation }}, T_{\text {Quiescent }}, \lambda\right)$ were adjusted to provide the optimal fit to the in vitro results. The optimal solution was identified by minimizing the Earth Mover's Distance [32] between the two histograms. We are also currently conducting a series of parametric studies in order to explore the influence that each component of cell behavior has on overall aggregation dynamics. Figure 6 contains some of our initial results. Here, we have significantly decreased and increased each cell's response to the chemoattractant gradient. It can be seen that slower cells (Left histogram) formed mostly smaller aggregates, yielding many more single cells. Faster cells (Right histogram) aggregated more aggressively and formed fewer smaller aggregates and more numerous larger aggregates.

\section{Conclusion}

We have described a software system that simulates chemotaxis-based cell aggregation in 2D. The model implemented within the system consists of such cell behaviors as chemical diffusion/detection, motility, proliferation, adhesion and life cycle stages. We have shown that the system is capable of performing a number of useful in silico experiments, including fine-tuning the model parameters to reproduce in vitro $\mathrm{PC} 12$ cell aggregation experiments and parametric studies that demonstrate the affect that each of the model's components have on cell aggregation dynamics. Future work will include extending the model and system to $3 \mathrm{D}$, and utilizing the system to study tumor growth.

This research has been funded by NSF Grant \# CCF-0636323 and NASA Grant \# NNJ04HC81G. 

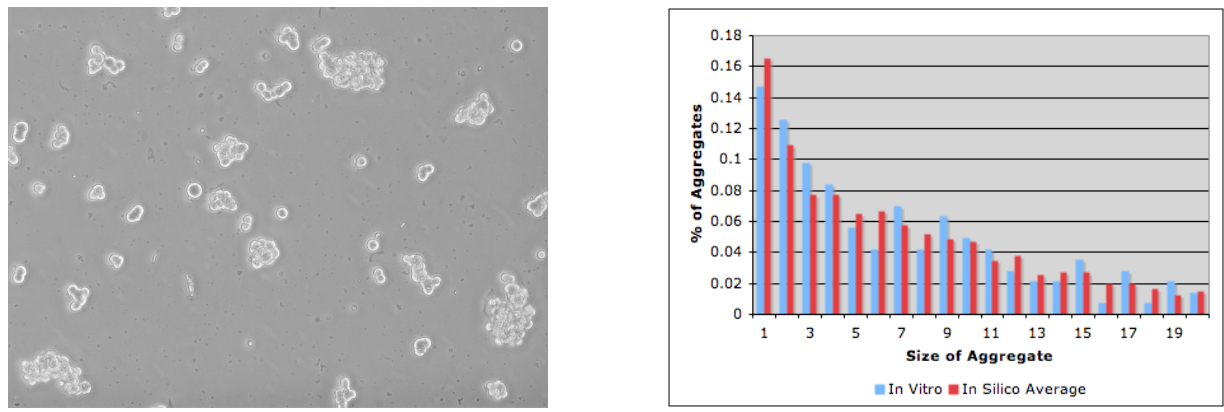

Fig. 5. Left: Image taken from an in vitro PC12 aggregation experiment after 24 hours. Right: Histogram comparing aggregate size distributions from an in vitro PC12 aggregation experiment (blue bars) and an optimized in silico cell aggregation experiment.
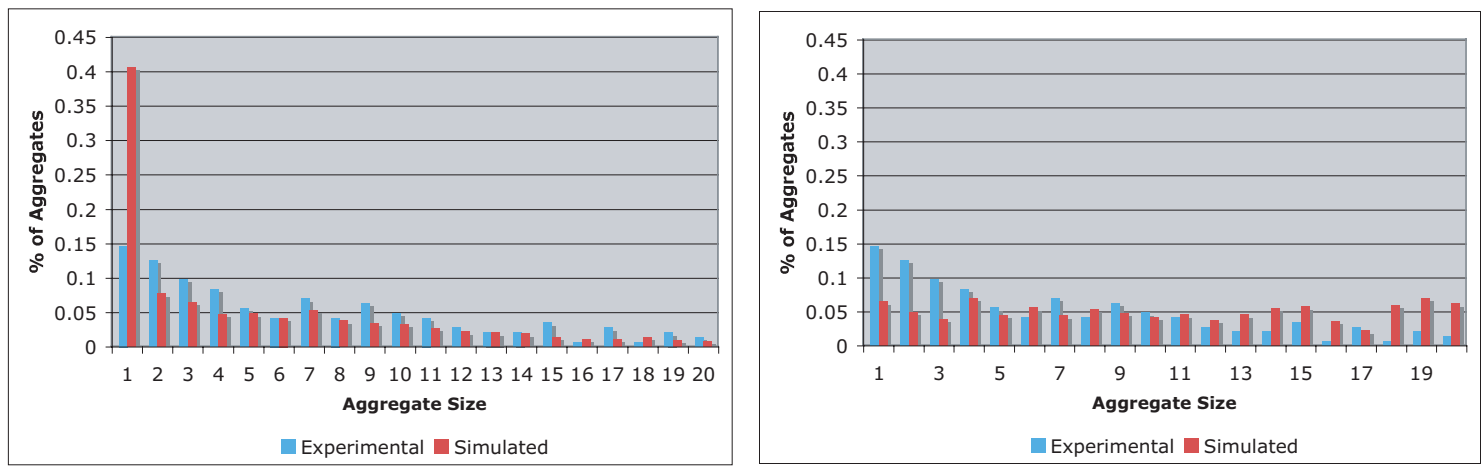

Fig. 6. Effect of chemoattractant gradient response on aggregation. Slower cells $(\lambda=0.01)$ (left) form fewer and smaller aggregates while faster cells $(\lambda=0.1)$ (right) form larger aggregates.

\section{References}

1. Fernandis, A., Prasad, A., Band, H., Klösel, R., Ganju, R.: Regulation of CXCR4-mediated chemotaxis and chemoinvasion of breast cancer cells. Oncogene 23(1) (2004) 157-167

2. Olson, M., Ford, R., Smith, J., Fernandez, E.: Mathematical modeling of chemotactic bacterial transport through a two-dimensional heterogeneous porous medium. Bioremediation Journal 10 (2006) 13-23

3. Graner, F., Glazier, J.A.: Simulation of biological cell sorting using a two-dimensional extended potts model. Physical Review Letters 69 (1992) 2013-2016

4. Chaturvedi, R., Huang, C., Kazmierczak, B., Schneider, T., Izaguirre, J.A., Newman, S.A., Glazier, J.A., Alber, M.: On multiscale approaches to three-dimensional modeling of morphogenesis. Journal of the Royal Society 2 (2005) 237-253

5. Glazier, J.A., Graner, F.: Simulation of the differential adhesion driven rearrangement of biological cells. Physical Review E 47 (1993) 2128-2154

6. Jiang, Y., Pjesivac-Grbovic, J., Cantrell, C., Freyer, J.: A multiscale model for avascular tumor growth. Biophysical Journal 89 (2005) 3884-3894

7. Merks, R.M.H., Glazier, J.A.: A cell-centered approach to developmental biology. Physica A 352 (2005) $113-130$

8. Hogeweg, P.: Evolving mechanisms of morphogenesis: on the interplay between differential adhesion and cell differentiation. Journal of Theoretical Biology 203 (2000) 317-333 
9. Hogeweg, P.: Computing an organism: on the interface between informatic and dynamic processes. Biosystems 64 (2002) 97-109

10. Tozeren, A., Coward, C.W., Petushi, S.P.: Origins and evolution of cell phenotypes in breast tumors. Journal of Theoretical Biology 233 (2005) 43-54

11. Ilachinski, A.: Cellular Automata : A Discrete Universe. World Scientific, Singapore (2001)

12. Wolfram, S.: Cellular automata as models of complexity. Nature 311 (1984) 419-424

13. Dormann, S., Deutsch, A.: Modeling of self-organized avascular tumor growth with a hybrid cellular automaton. In Silico Biology 2(3) (2002) 393-406

14. Patel, A.A., Gawlinski, E.T., Lemieux, S.K., Gatenby, R.A.: A cellular automation model of early tumor growth and invasion: the effects of native tissue vascularity and increase in anaerobic tissue metabolism. Journal of Theoretical Biology 213 (2001) 315-331

15. Longo, D., Peirce, S.M., Skalak, T.C., Davidson, L., Marsden, M., Dzamba, B., Simone, D.W.D.: Multicellular computer simulation of morphogenesis: blastocoel roof thinning and matrix assembly in xenopus laevis. Developmental Biology 271 (2004) 210-222

16. Bagchi, P., Johnson, P.C., Popel, A.S.: Computational fluid dynamic simulation of aggregation of deformable cells in a shear flow. Transactions of the ASME 127 (2005) 1070

17. Song, H., Jain, S.K., Enmon, R.M., O'Connor, K.C.: Restructuring dynamics of DU 145 and lncap prostate cancer spheroids. In Vitro Cellular and Developmental Biology-Animal 40 (2004) 262-267

18. Ascencio, S.F., Meana, H.P., Miyatake, M.N.: Two and three dimensional computer simulation of cancer growth. Proc. XXI Int. Conference of the Chilean Computer Science Society (2001) 73-79

19. Fleischer, K.W., Barr, A.H.: A simulation testbed for the study of multicellular development: the multiple mechanisms of morphogenesis. Artificial Life III (1994) 389-408

20. Fleischer, K.W.: Investigations with a multicellular developmental model. Artificial Life V (1996) 229-236

21. Jabbarzadeh, E., Abrams, C.F.: Chemotaxis and random motility in unsteady chemoattractant fields: A computational study. Journal of Theoretical Biology 235 (2005) 221-232

22. Palsson, E., Othmer, H.: A model for individual and collective cell movement in dictyostelium discoideum. Proceedings of the National Academy of Science USA 97 (2000) 10448-10453

23. N'Dri, N.A., Shyy, W., Tran-Son-Tay: Computational modeling of cell adhesion and movement using a continuum-kinetics approach. Biophysical Journal 85 (2003) 2273-2286

24. Izaguirre, J.A., Chaturvedi, R., Huang, C., Cickovski, T., Coffland, J., Thomas, G., Forgacs, G., Alber, M., Newman, S., Glazier, J.A.: Compucell, a multi-model framework for simulations of morphogenesis. Bioinformatics 20 (2004) 1129-1137

25. Cickovski, T., et al.: A framework for three-dimensional simulation of morphogenesis. IEEE/ACM Transactions on Computational Biology and Bioinformatics 2 (2005) 273-288

26. Alberts, B., Bray, D., Hopkin, K., Johnson, A., Lewis, J., Raff, M., Roberts, K., Walter, P.: Essential Cell Biology. 2nd edn. Garland Publishing (2003)

27. Savinell, J.M., Lee, G.M., Palsson, B.O., Arbor, A.: On the orders of magnitude of epigenic dynamics and monoclonal antibody production. Bioprocess Engineering 4 (1989) 231-234

28. Serini, G., et al.: Modeling the early stages of vacular network assembly. The EMBO Journal 22(8) (2003) 1771-1779

29. Watanabe, O., Torda, M., Meldolesi, J.: The effect of $\alpha$-latrotoxin on the neurosecretory PC12 cell line: Electron microscopy and cytotoxicity studies. Neuroscience 10(3) (1983) 1011-1024

30. Hirata, Y., Adachi, K., Kiuchi, K.: Activation of JNK pathway and induction of apoptosis by manganese in PC12 cells. Journal of Neurochemistry 71 (1998) 1607-1615

31. Manley, P., Lelkes, P.: A novel real-time system to monitor cell aggregation and trajectories in rotating wall vessel bioreactors. Journal of Biotechnology 125(3) (2006) 416-424

32. Rubner, Y., Tomasi, C., Guibas, L.: The earth mover's distance as a metric for image retrieval. International Journal of Computer Vision 40(2) (2000) 99-121 


\section{A Appendix: Algorithm Pseudocode}
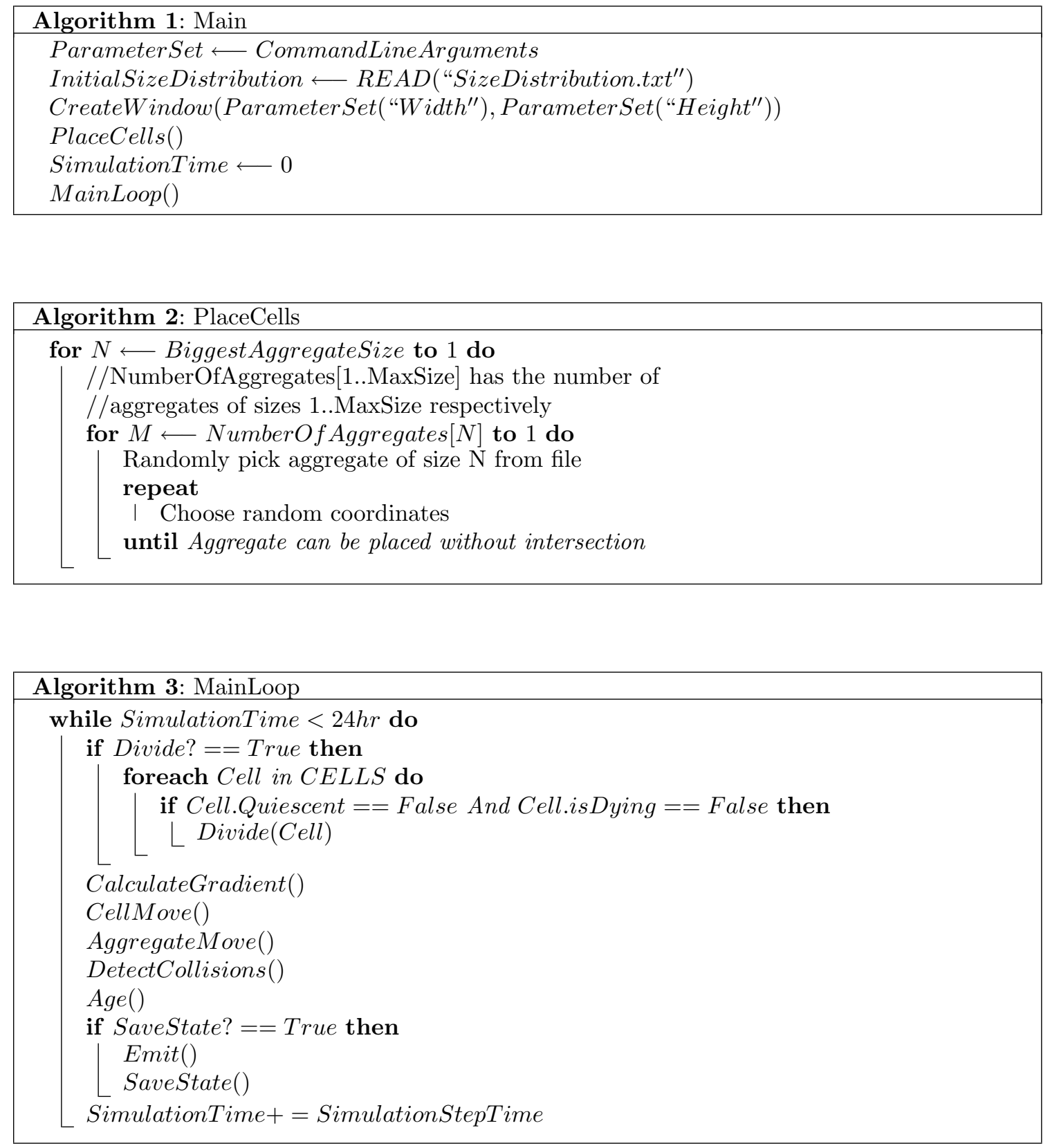


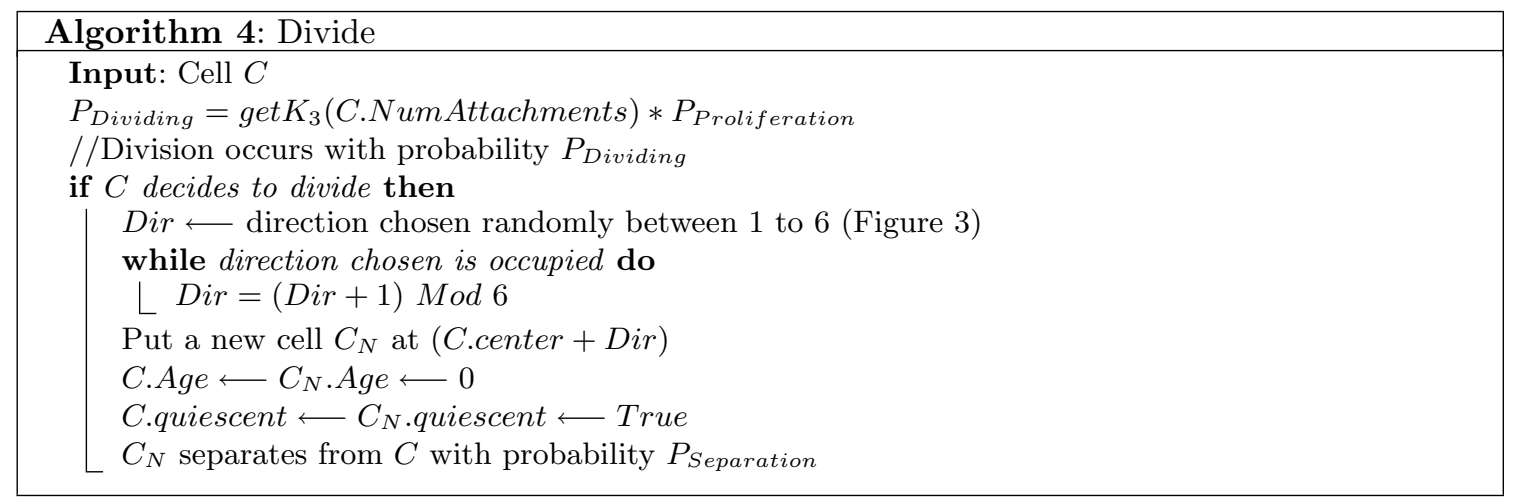

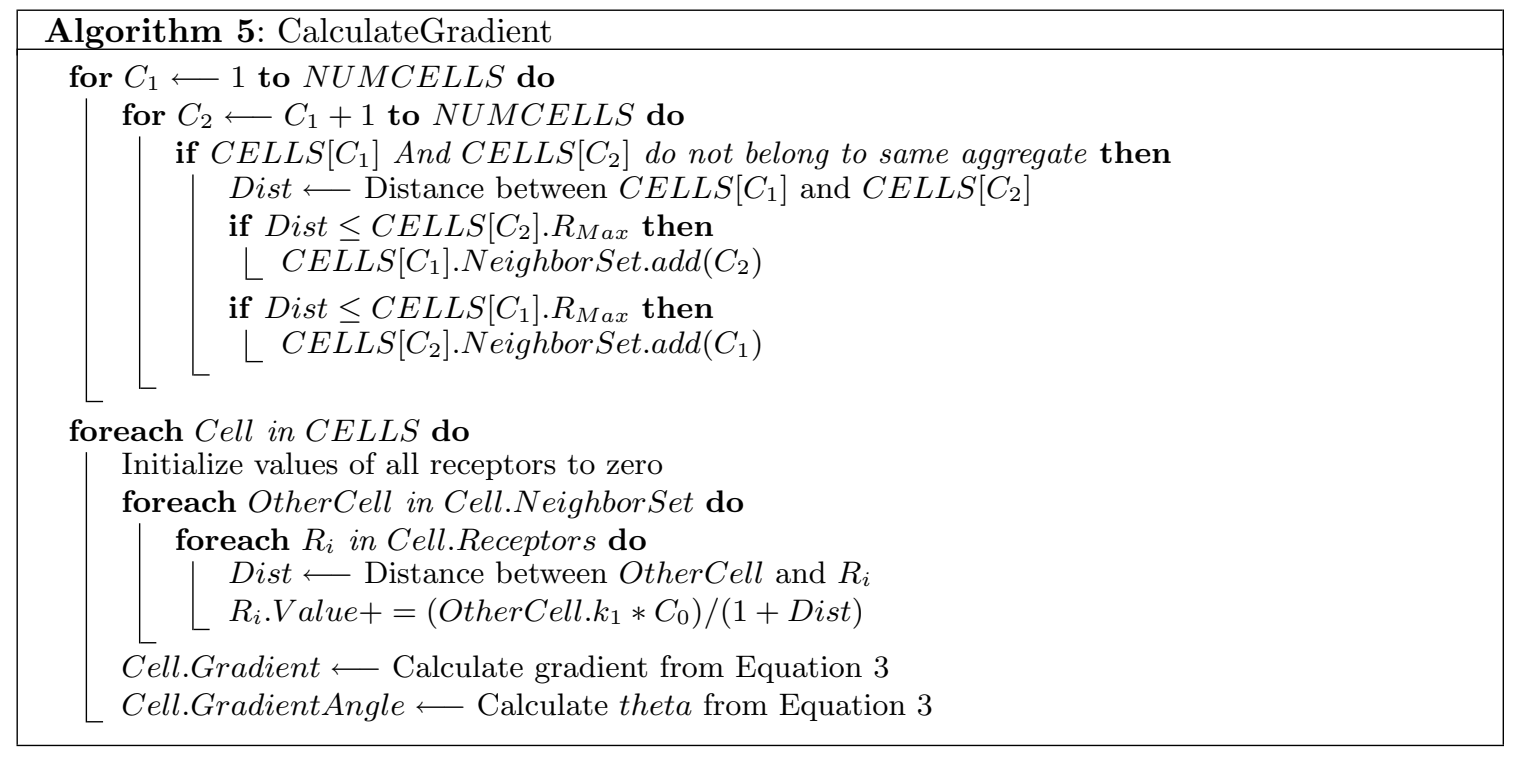



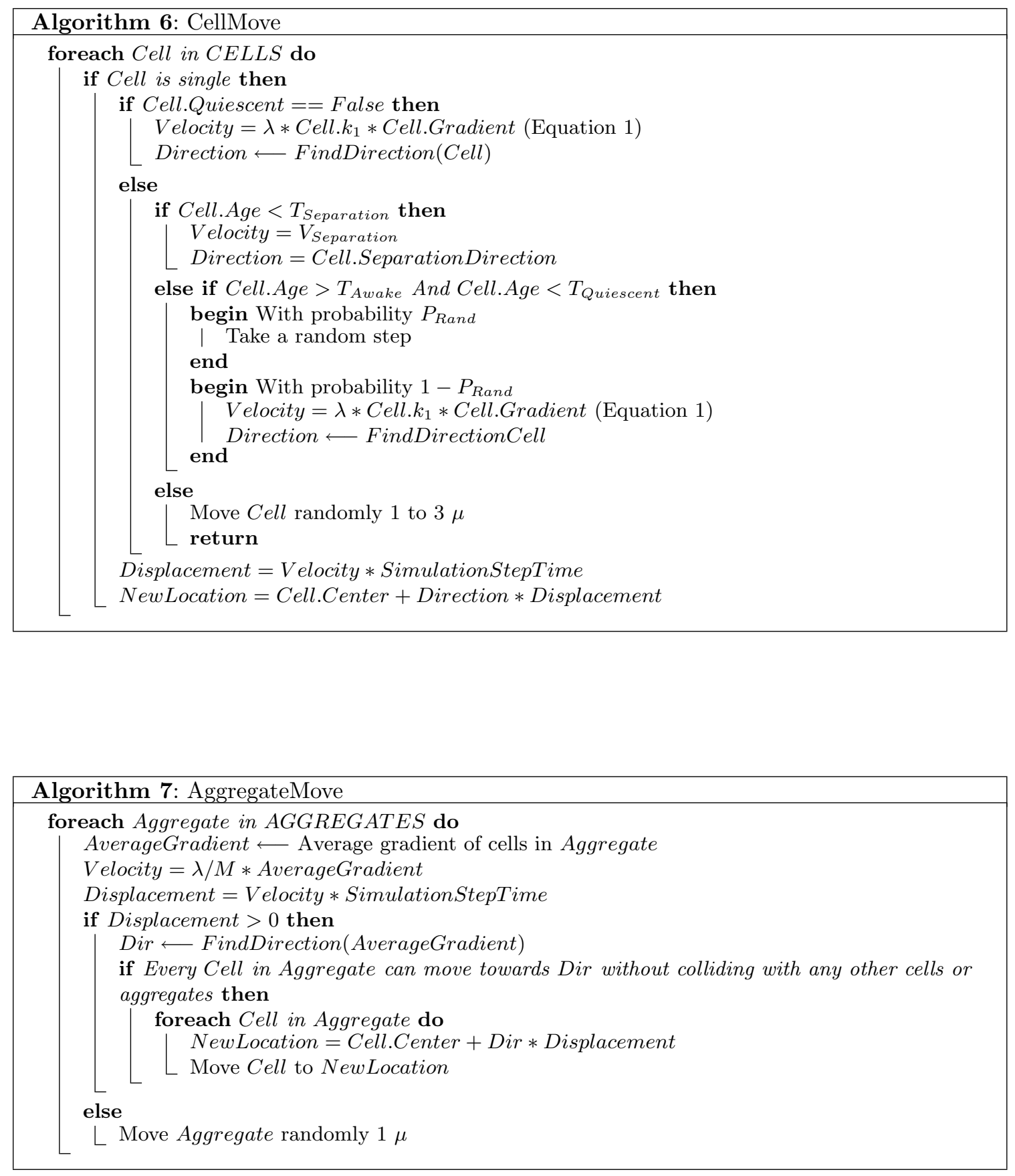

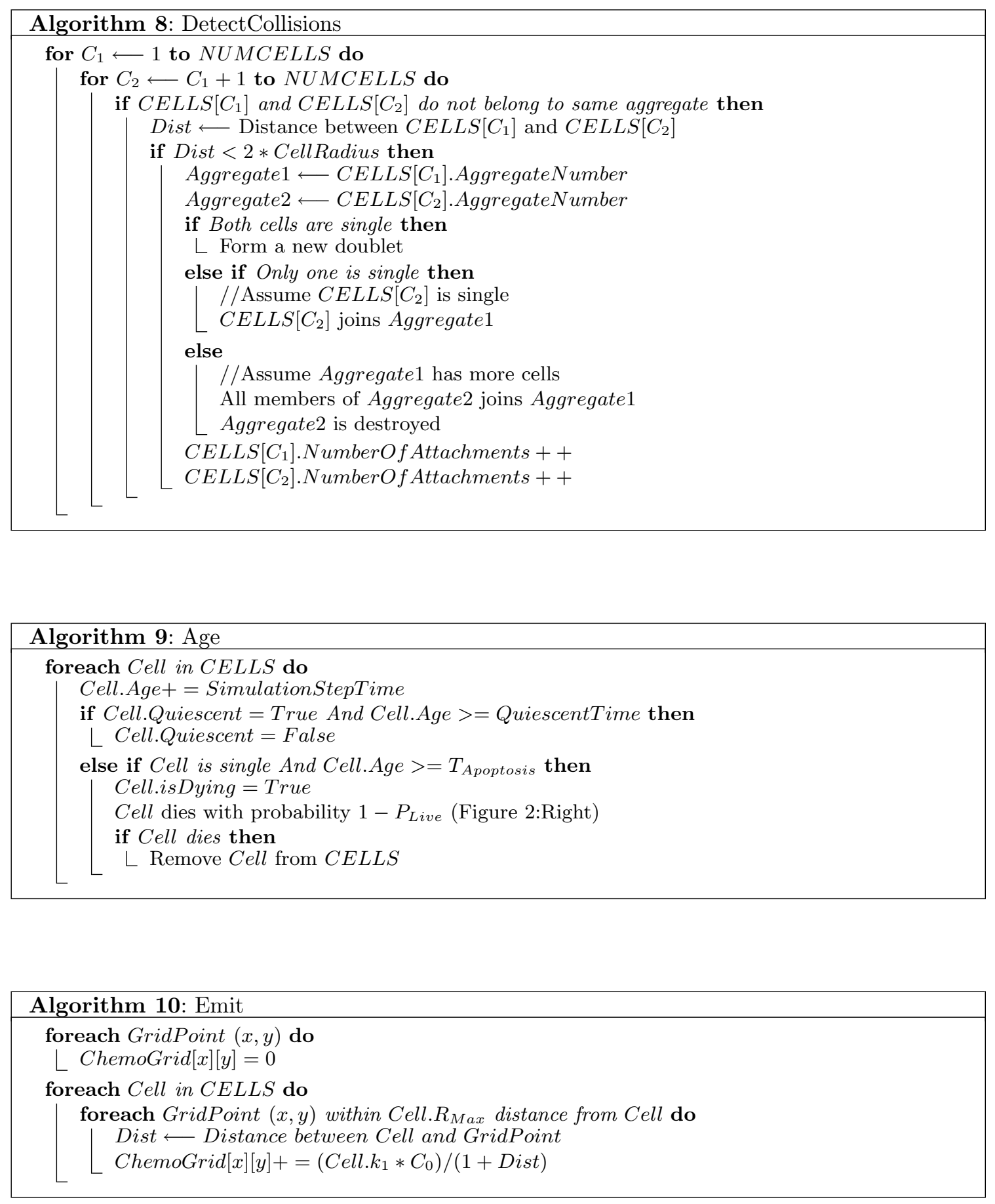